bioRxiv preprint doi: https://doi.org/10.1101/2020.05.22.110403; this version posted May 22, 2020. The copyright holder for this preprint (which

was not certified by peer review) is the author/funder, who has granted bioRxiv a license to display the preprint in perpetuity. It is made available under aCC-BY 4.0 International license.

1 Long Title: Methodological Issues with Search in MEDLINE: A Longitudinal Query Analysis

2 Short Title: Methodological Issues with Search in MEDLINE

3 C. Sean Burns ${ }^{1}$, Tyler Nix², Robert M. Shapiro II ${ }^{1}$, Jeffrey T. Huber ${ }^{1}$

$4{ }^{1}$ University of Kentucky, School of Information Science

52 Taubman Health Sciences Library, University of Michigan 
6 Abstract: This study compares the results of data collected from a longitudinal query analysis of

7 the MEDLINE database hosted on multiple platforms that include PubMed, EBSCOHost, Ovid,

8 ProQuest, and Web of Science in order to identify variations among the search results on the

9 platforms after controlling for search query syntax. We devised twenty-nine sets of search

10 queries comprised of five queries per set to search against the five MEDLINE database

11 platforms. We ran our queries monthly for a year and collected search result count data to

12 observe changes. We found that search results vary considerably depending on MEDLINE

13 platform, both within sets and across time. The variation is due to trends in scholarly publication

14 that include publishing online first versus publishing in journal issues, which leads to metadata

15 differences in the bibliographic record; to differences in the level of specificity among search

16 fields provided by the platforms; to database integrity issues that lead to large fluctuations in

17 monthly search results based on the same query; and to database currency issues that arise due to

18 when each platform updates its MEDLINE file. Specific bibliographic databases, like PubMed

19 and MEDLINE, are used to inform clinical decision-making, create systematic reviews, and

20 construct knowledge bases for clinical decision support systems. Since they serve as essential

21 information retrieval and discovery tools that help identify and collect research data and are used

22 in a broad range of fields and as the basis of multiple research designs, this study should help

23 clinicians, researcher, librarians, informationalists, and others understand how these platforms

24 differ and inform future work in their standardization. 


\section{Methodological Issues with Search in MEDLINE: A 26 Longitudinal Query Analysis}

\section{Introduction}

28 Bibliographic databases are used to identify and collect research data, and therefore function as

scientific instruments [1,2]. Studies that rely on these instruments include research on

information literacy, bibliometrics/scientometrics, information seeking, systematic reviews,

31 literature reviews, and meta-analyses [3]. These systems, in particular, PubMed and MEDLINE, are also used to inform clinical decision-making in the health professions [4] and construct knowledge bases for clinical decision support systems [5].

Research on search queries that inform the development of bibliographic databases or on how queries influence information retrieval sets were once common lines of inquiry [6] but these have subsided in recent decades [7]. Search query research has largely shifted away from a Boolean model of information retrieval and has focused on ranked-based keyword systems [8] or on database coverage [9-12].

Researchers, librarians, information scientists, and others rely on bibliographic databases to conduct research, to instruct future information and other professionals how to conduct literature

41 searches, and to assist those with information needs to locate and access literature [13-16].

42 Furthermore, an entire bibliographic universe exists based on bibliographic control that includes

43 standardized rules for description, authority files, controlled vocabularies, and taxonomies to

44 help make searching for information more precise or comprehensive $[17,18]$.

45 Fine control over bibliographic search and the documentation of search strategies, which are

46 often reported in systematic review research, should allow for the replication and reproduction of

47 searches. In the broader scientific community, the replication and reproduction of research, or 
48 lack thereof, has garnered increased attention recently [19,20]. Additional scrutiny has been

49 given to the replication of prior studies [21]. This is true for systematic reviews and other

50 research that relies on citation or bibliographic records, but in this domain, the evaluation of

51 scientific rigor is centered around the reproducibility of search strategies. The Preferred

52 Reporting Items for Systematic Reviews and Meta-Analyses (PRISMA) Guidelines [22] and the

53 Cochrane Handbook for Systematic Reviews of Interventions provide examples of how scholars

54 have recognized the need for the systematic reporting of methods and the organization of review

55 research [23].

56 Unlike general search engines, bibliographic databases, such as those available on EBSCOhost,

57 ProQuest, Web of Science, Scopus, Ovid, and others rely on structured bibliographic records

58 instead of full text sources to create search indexes. These bibliographic records contain fields

59 we take as meaningful for providing discovery and access, such as author name fields, document

60 title fields, publication title fields, and date of publication fields [17]. In some specialized

61 databases, these records may be supported by controlled terminologies that are database specific

62 or are based on standard knowledge classification efforts, such as the Medical Subject Headings

63 (MeSH) or the Library of Congress Subject Headings (LCSH).

64 Controlled vocabularies, thesauri, and taxonomies are meant to provide users with a high level of

65 control over the search and retrieval process [24,25]. Some thesauri systems are available across

66 multiple platforms or interfaces [26]. For example, the ERIC thesaurus is freely available at the

67 U.S. Department of Education's (DOE) ERIC digital library (eric.ed.gov) but also through

68 subscription-based platforms provided by EBSCOhost and ProQuest. Similarly, the MeSH

69 thesaurus is freely available on the U.S. National Library of Medicine's (NLM) digital library, 
70 PubMed, but can be used to search on platforms provided by EBSCOhost, Ovid ProQuest, Web

71 of Science, and others.

72 Commercial information service companies presumably provide their own access points to

73 bibliographic data from ERIC and PubMed, and the corresponding thesauri, on their own

74 platforms in order to add value above and beyond what the original database providers, like

75 NLM or DOE, have created and provided access to. The added value may be based upon the

76 provider's unique user interface, its search technologies, its ability to link to library collections

77 via proxy software, its additional database content, or its ability to search against multiple

78 databases on a specific platform in single search sessions.

79 However, adding value entails some differentiation from the original system [26,27] that may

80 introduce variation in search results. For example, the MEDLINE database accessed through

81 PubMed is defined by the list of publication titles it indexes, the data structure of its

82 bibliographic records, the application of $\mathrm{MeSH}$ to the bibliographic records in those records, and

83 the search technologies it implements. When a commercial information service provider also

84 provides access to MEDLINE, it uses that base system, but also differentiates itself from

85 PubMed by providing a different interface, search fields, search operators, and other

86 technologies.

87 This differentiation among database platforms has long been recognized as important in the

88 systematic review literature in the biomedical sciences, and because of this, the forthcoming

89 PRISMA-S Search Reporting Extension recommends that systematic reviewers report which

90 platform, interface, or vendor is used for each database searched [28]. However, the implications

91 of this differentiation across platforms, with respect to how bibliographic records are queried, are

92 not well understood [29,30]. For example, even though PubMed/MEDLINE, 
93 ProQuest/MEDLINE, EBSCOhost/MEDLINE, Ovid/MEDLINE, and Web of

94 Science/MEDLINE are presumably built on the same MEDLINE data file, it is not fully known

95 how the alterations that are made by these vendors impact search and retrieval on their respective

96 platforms. Even when there is some transparency, such as with PubMed [31], these systems are

97 complicated and differences with other systems are not well understood.

98 Although the choice of database systems impacts potential source coverage and search methods,

99 it is not known how searching the dame database (e.g., MEDLINE) on different platforms might

100 affect source coverage. If searchers used too few databases to conduct literature searches [32-

101 34], then they may miss relevant studies [10,35]. This is especially important in cases where data

102 from past research is collected, analyzed, and synthesized based on published and/or gray

103 literature, such as in systematic reviews or meta-analyses [11,36]. Studies have also highlighted

104 problems in the reporting of search strategies, highlighting incomplete details necessary for

105 others to investigate the quality of a search strategy [37], but this assumes consistency between

106 platforms that provide access to the same database: for example, that using MEDLINE on

107 PubMed is equivalent to using MEDLINE on Ovid, EBSCOhost, ProQuest, or Web of Science.

108 This may have ramifications for those researchers leading clinical trials or conducting bench-side

109 research, and who have to rely on published literature and conduct intensive literature searches

110 when systematic reviews on their topic are not available.

111 Even if search sessions are methodical and well documented, database systems often operate as

112 black boxes (i.e., the technology is not well documented) and it becomes only possible to infer

113 how different systems operate by comparing multiple implementations [38]. Little is thus known

114 about what actual principles are applied by database vendors in indexing bibliographic records or

115 what specific sets of algorithms are used to rank results when sorted by system-defined 
116 relevance. This is commonly known problem among commercial search engines, but it is also

117 problematic in bibliographic databases purchased by libraries [39,40].

118 Interface, indexing, and retrieval differences also impact reproducibility and replication, which

119 are important aspects of the scientific process, evidence-based medicine, and the creation of

120 systematic reviews [33,41-44]. Although the NLM maintains the MEDLINE records and

121 provides free (federally subsidized) access to them through the PubMed website, they also

122 license these records to database vendors to host on their own platforms. Furthermore, although

123 these systems operate from the same MEDLINE data file, database vendors apply their own

124 indexing technologies and their own search interfaces, and it is possible that these alterations

125 influence different search behaviors and retrieval sets $[45,46]$. This may be problematic if

126 platform differences are not commonly understood, communicated in vendor reports, or among

127 research team members using them, and if the separate platforms are unable to replicate results

128 based on the same data files that are used across them.

129 While some studies have included queries that were designed to be reproducible across systems

130 [35], most studies compare queries across systems by evaluating recall and precision on the

131 retrieval sets in these systems [47-49]. However, the focus is not often on the query syntax used

132 even though this has been highlighted as an important problem. One study investigated variations

133 among different interfaces to the Cumulative Index for Nursing and Allied Health Literature

134 (CINAHL) database, and reported reproducible search strategies except for queries that

135 contained subject-keyword terms [29]. In our prior paper (XXXX) we found that queries

136 searched in MEDLINE across different platforms resulted in search result discrepancies after

137 controlling for the search query. This paper extends upon that work with longitudinal data. Here

138 we ask the following research questions: 
1. How do search results among MEDLINE-based bibliographic database platforms vary over time after controlling for search query syntax?

2. What explains the variance among search results among MEDLINE-based bibliographic database platforms after controlling for search query syntax?

143 To answer these questions, our analytical framework is based on the concepts of methods and

144 results reproducibility [50]. Methods reproducibility is "the ability to implement, as exactly as

145 possible, the experimental and computational procedures, with the same data and tools, to obtain

146 the same results" and results reproducibility is "the production of corroborating results in a new

147 study, having followed the same experimental methods (A New Lexicon for Research

148 Reproducibility section, para. 2). We do not apply the concept of inferential reproducibility in

149 this paper since this pertains to the conclusions that a study makes based on the reproduced

150 methods, and this would largely be applicable if we investigated the relevance of the results

151 based on an information need rather than, as we do, focus solely on the reproducible sets of

152 search queries and the records produced by executing those queries.

\section{Materials and Methods}

154 We conducted a longitudinal study (October 2018-September 2019) on five MEDLINE-based

155 platforms after two pilot runs in August and September 2018. The five platforms include what is

156 now legacy PubMed/MEDLINE (PMML), which is undergoing an interface update that applies

157 new search algorithms [51], ProQuest/MEDLINE (PQML), EBSCOhost/MEDLINE (EHML),

158 Web of Science/MEDLINE (WSML), and Ovid/MEDLINE (OML). The data is based on search

159 result counts for each query in each set and was collected once per month at the mid-point of

160 each month. Twenty-nine sets of search queries were created to search these platforms with each

161 set containing five queries for the respective platforms and for a total of 145 searches per month. 
162 The search queries, tested in the pilot studies, were designed to be semantically and logically

163 equivalent to each other on a per set basis. Differences between queries within sets were made

164 only to adhere to the query syntax required for each platform. Table 1 provides an example

165 search set (\#010) for October 2018. Each of the queries in this specific set were designed to

166 search for the MeSH term dementia, which has two three numbers (MeSH branches), to explode

167 the term, and to limit results by publication date from 1950 to 2015 . The last column reports the

168 number of records that were retrieved for each of the platforms for the month and year that data

169 was collected. 
170 Table 1. Example set of search queries across the five MEDLINE platforms

\begin{tabular}{|l|l|l|}
\hline Platform & Search Set \#010 & $10-2018$ \\
\hline PMML & "dementia" [MH] AND 1950:2015 [DP] & 134217 \\
\hline PQML & MESH.EXACT.EXPLODE ( "dementia") AND YR (1950-2015) & 132593 \\
\hline EHML & MH("dementia+") AND YR 1950-2015 & 132599 \\
\hline WSML & MH:exp=("dementia") AND PY=(1950-2015) & 132590 \\
\hline OML & 1. EXP dementia/ 2. limit 1 to YR=1950-2015 & 132593 \\
\hline
\end{tabular}

171 Our 29 sets of search queries were designed to test the basic functionality of the five platforms

172 in order to compare the results without introducing too much complexity. Therefore, the queries

173 were not designed to test user relevance or user needs, which may range from simple searches to

174 complex, multi-part queries designed for systematic review research. Rather, our queries were

175 designed to test basic functionality with respect to searches that contained some part or

176 combination of the following search fields:

177 - Keywords

- $\quad$ Specific fields

- MeSH terms with more than one tree number

- $\mathrm{MeSH}$ terms that were exploded

182 Nine of our search sets included publication date ranges. We added date ranges to test whether

183 search result counts would remain frozen after the end of the publication date and to add an

184 additional control that would help explain differences between the platforms. Most queries also

185 included at least one Boolean operator. All search queries and search result counts are included

186 in the data repository, but Table 2 describes the generalized parameters for each of the 29 sets. 
187 Table 2. Generalized search parameters for all 29 sets of search queries

\begin{tabular}{|c|c|c|c|c|c|c|c|c|c|}
\hline $\begin{array}{l}\text { Search Set } \\
\text { Number }\end{array}$ & Keyword & FieldSpecific & MeSH & Branches & PubDate & Explode & AND & OR & NOT \\
\hline 001 & 1 & 0 & 0 & 0 & 0 & 0 & 0 & 0 & 0 \\
\hline 002 & 0 & 0 & 1 & 1 & 0 & 0 & 0 & 0 & 0 \\
\hline 003 & 1 & 0 & 0 & 0 & 1 & 0 & 1 & 0 & 0 \\
\hline 004 & 0 & 0 & 1 & 1 & 1 & 0 & 1 & 0 & 0 \\
\hline 005 & 1 & 0 & 1 & 1 & 1 & 0 & 2 & 0 & 0 \\
\hline 006 & 0 & 0 & 1 & 2 & 1 & 0 & 1 & 0 & 0 \\
\hline 007 & 1 & 0 & 1 & 2 & 1 & 0 & 2 & 0 & 0 \\
\hline 008 & 0 & 0 & 1 & 1 & 1 & 1 & 1 & 0 & 0 \\
\hline 009 & 1 & 0 & 1 & 1 & 1 & 1 & 2 & 0 & 0 \\
\hline 010 & 0 & 0 & 1 & 2 & 1 & 1 & 1 & 0 & 0 \\
\hline 011 & 1 & 0 & 1 & 2 & 1 & 1 & 2 & 0 & 0 \\
\hline 012 & 0 & 0 & 2 & 2 & 0 & 2 & 0 & 0 & 1 \\
\hline 013 & 0 & 1 & 2 & 2 & 0 & 2 & 1 & 0 & 1 \\
\hline 014 & 0 & 2 & 2 & 2 & 0 & 2 & 1 & 0 & 2 \\
\hline 015 & 0 & 1 & 2 & 2 & 0 & 2 & 0 & 0 & 2 \\
\hline 016 & 0 & 1 & 2 & 2 & 0 & 2 & 1 & 0 & 1 \\
\hline 017 & 0 & 1 & 2 & 2 & 0 & 2 & 1 & 0 & 1 \\
\hline 018 & 0 & 1 & 0 & 0 & 0 & 0 & 0 & 0 & 0 \\
\hline 019 & 0 & 2 & 1 & 1 & 0 & 0 & 1 & 1 & 0 \\
\hline
\end{tabular}




\begin{tabular}{|l|r|r|r|r|r|r|r|r|r|}
\hline 020 & 0 & 4 & 0 & 0 & 0 & 0 & 1 & 2 & 0 \\
\hline 021 & 1 & 2 & 0 & 0 & 0 & 0 & 1 & 1 & 0 \\
\hline 022 & 0 & 0 & 1 & 2 & 0 & 0 & 0 & 0 & 0 \\
\hline 023 & 0 & 0 & 1 & 2 & 0 & 1 & 0 & 0 & 0 \\
\hline 024 & 0 & 0 & 2 & 6 & 0 & 0 & 1 & 0 & 0 \\
\hline 025 & 0 & 0 & 2 & 6 & 0 & 1 & 1 & 0 & 0 \\
\hline 026 & 0 & 0 & 2 & 3 & 0 & 0 & 0 & 0 & 1 \\
\hline 027 & 0 & 0 & 2 & 3 & 0 & 1 & 0 & 0 & 1 \\
\hline 028 & 0 & 0 & 2 & 3 & 0 & 2 & 1 & 0 & 0 \\
\hline 029 & 0 & 0 & 2 & 3 & 0 & 1 & 1 & 0 & 0 \\
\hline
\end{tabular}

Note: Column meanings: The Keyword column indicates how many keywords were used in the query, not counting field specific keywords, such as document title, journal title, or author name. The latter are counted in the FieldSpecific column, which indicates the number of field specific terms used in the query. The MeSH column indicates how many MeSH terms were used in the query. The Branches column indicates how many trees a MeSH term belongs to. The PubDate column is a binary column to indicate whether a query does not include a publication date (0) or includes a publication date (1). The Explode column indicates whether a MeSH term was not exploded (0), exploded (1), or in queries with multipe MeSH terms, at least one term was exploded and one was not (2). The $A N D, O R$, and NOT columns indicate a count of how many of these Boolean operators were used in the query. In legacy PubMed, queries require an 'and medline[sb]' tag in order to limit results to MEDLINE only and to exclude PubMed more broadly. These ANDs were not counted in this column. We did count ANDs when used to join terms or when including publication date ranges in our searches, even for Ovid/MEDLINE, even though Ovid/MEDLINE uses the limit operator and not technically the AND operator.

\section{Results}

201 Our query sets include 100 queries without publication date ranges and nine sets containing 45

202 queries with publication date ranges from 1950-2015. Thirty-nine of the publication date

204 indicating potential changes either in the bibliographic records nearly five years after the last

205 publication or potential changes with the search mechanisms used among the MEDLINE 
207 and in the following sections, we describe some major themes of these differences among these 208 platforms.

\section{Macro and Micro Views of the Data Reveal Different Trends}

210 A macro examination of the search sets restricted by publication date (search sets \#003-\#011)

211 mainly indicated that there were only substantial differences in total search result counts over

212 time between platforms within each set, for example, between WSML and PMML (Figure 1, top

213 left plot). This macro view would appear to indicate that although there are differences among

214 platforms, the comparable tends are reliably consistent across time on a per query, per platform

215 basis. However, a re-scaled, side-by-side comparison of result counts per platform indicates

216 more variation within the platforms themselves (Figures 2-4). This illustrates that platforms are

217 not internally reliable across time on a per query basis. Furthermore, there is also no apparent

218 difference in the growth trends between queries that are restricted by publication date and those

219 that are not restricted by publication date (Figure 5). Figure 5 illustrates the annual growth of

220 records over the year for sets with queries restricted by publication dates and sets of queries with

221 no publication date restrictions and shows that queries restricted by publication dates continue to

222 return new results years past the end of the publication date range.

223 [ Insert Figure 1 ]

224 Fig. 1. A macro scaled view of search result counts for eleven search sets restricted by

225 publication date. Each plot indicates a separate search set.

226 [ Insert Figures 2-4 ]

227 Fig. 2-4. A micro scaled-view of search result counts for eleven search sets restricted by

228 publication date. Each row indicates a search set. 
230 Fig. 5. A side-by-side comparison of the growth of search result counts for search sets that were

231 not limited by publication date (white bars, on the left) and search result counts for search sets

232 that were limited by publication date (black bars, on the right).

\section{Online-first and Print Publications Reduce Effectiveness of 234 Bibliographic Control}

235 To help identify an explanation for the changes in search results for queries that were restricted

236 by publication dates, we compared two query sets, query \#002 and query \#004, which were both

237 designed to search for a single MeSH term ("neoplasms"), non-exploding, but differed in that

238 query \#004 is publication date restricted. Hypothetically, searches for MeSH terms should not be

239 impacted by changes in search mechanisms since the search process for a controlled term is

240 based on whether a record contains the term or not. The grand median change over the year in

241 search result counts for query \#002 among all five platforms was 16,551 records (max: 17102;

242 min: 15933), indicating the hypothetical annual growth in literature attached to this term since

243 this query was not restricted by publication date. The grand median change in search results for

244 query \#004 among all five platforms was 17 records (max: 70; min: 8) for query \#004. Since this

245 query set was restricted by publication date, this indicates hypothetical changes that are not

246 related to literature growth but to changes in either the database search indexes or the

247 bibliographic records, four years after publication.

248 Furthermore, since all platforms reported different search result numbers in this query set, this

249 indicates that the five platforms are indexing different versions of the same MEDLINE file, or

250 that the platforms index the basic MEDLINE file differently based not on the MeSH term but on

251 the publication date field. To test this, we traced a record from the \#004 query results. The record 
252 we investigated was chosen because it was part of the retrieval set for a query that was limited by

253 the publication date to 2015 but which the record indicated it was published in 2019.

254 According to PubMed documentation, the default publication date field [DP] or [PDAT] includes

255 the date of publication for either the electronic or print version [52]. An investigation of the

256 chosen record from the search results for \#004 in the PMML set [53] shows a bibliographic

257 record with a long publication history. The PubMed record indicates that the record was added to

258 PubMed in 2015 but not entered into MEDLINE proper until 2019 (See:

259 https://www.ncbi.nlm.nih.gov/pubmed/26700484?report=medline\&format=text). On the journal

260 (BMJ) web page, there are two versions of the article - an "Online First" version for the article

261 that was issued in 2015 (See: https://spcare.bmj.com/content/early/2015/12/23/bmjspcare-2014-

262 000835.info?versioned=true) and an "online issue" version of the article that was issued in 2019

263 (See: https://spcare.bmj.com/content/9/1/67.info). The journal article's publication history on its

264 web page states that the 2015 version of the article is the online first article, and the 2019 version

265 is the publication date for when the article was assigned to a volume and issue and when it then

266 appeared in print and was added to MEDLINE based on the bibliographic data attached to the

2672019 version. On the journal's site, there are thus two versions of this article. On PubMed, there

268 is one record for this article with two publication dates because of versioning.

269 The above record indicates problems with bibliographic control and dependency on journals to

270 maintain bibliographic records that are complicated by two sequences of publications: online

271 publications that precede print publications for the same article, or Online First publications that

272 preceded publications that are attached to volume and issue numbers. The latter are further

273 complicated by the versioning of articles based on publication history and that include versions

274 prior to their official publication dates when they are assigned to volume and issue numbers and 
275 then added to MEDLINE. This problem with bibliographic control impacts search results across

276 the platforms. The BMJ article described above does not appear in the search results among the

277 other four MEDLINE platforms for query set \#004. This confirms that the PMML platform

278 captures the electronic publication date by default even though the record was not entered into

279 MEDLINE proper until the print publication date which, in this case, was four years after the

280 electronic publication date and even though the query was restricted to MEDLINE. Neither

281 PQML, EHML, WSML, nor OML behave in this way. PQML and WSML only offer the ability

282 to search by a single publication date limit, which seems to be defined by the e-publication date,

283 and these platforms do not offer the ability to search by other date fields. EHML and OML offer

284 more control over a variety of date fields but apparently the default publication date field is not

285 inclusive of print publication dates between these two platforms, like it is in PMML.

\section{Reproducible With Specific Field Searches}

287 We found that the sets of queries that returned nearly equivalent search result counts were

288 queries that included additional specificity, regardless if the queries were restricted by

289 publication date. Query \#013 included two MeSH terms, the first one non-exploded and the

290 second one exploded, that were connected by one Boolean NOT, plus one document title term.

291 All five platforms returned results that were within a range of 3 records, taking into account the

292 range of results per platform and then among platforms over the annual period of data collection.

293 This relative consistency across platforms was found in other search sets that included additional,

294 specific field searches. For example, query set \#018 performed a single author search for an

295 author who was chosen because they had published in journals indexed by MEDLINE during the

296 middle parts of the 20th century. The range of records that were returned varied over the months

297 and numbered within a range of 15 records among the others. However, when a MeSH term was 
298 added to the author name search (search set \#017), chosen because the author had published

299 papers that had been indexed with the specific MeSH term ("neoplasms"), all five platforms

300 returned the same count of records for all twelve months of data collection.

\section{Database Integrity Fails}

302 Aside from issues with bibliographic control due to online versioning, and with differences in

303 indexing, several of the platforms returned results that appear as outliers compared to the others

304 within a set. The query in search set \#008 included one MeSH term, on a single branch,

305 exploded, with a publication date restriction. PubMed/MEDLINE returned a range of 219

306 additional records across the months. ProQuest/MEDLINE returned a range of 211 records, and

307 Ovid/MEDLINE returned a range of 438 records. However, EBSCOhost/MEDLINE returned a

308 range of 2108 records, and although Web of Science/MEDLINE returned a range of only 11

309 records for the time period, it also returned an average of 2491138 fewer records than

310 PubMed/MEDLINE. We could find no discernible reason for this discrepancy. Search set \#010

311 and search set \#023 both included a single MeSH term, two branches, exploded, and although

312 search result counts were different among these platforms within these sets, the differences were

313 not as extreme, perhaps then indicating a problem with how Web of Science/MEDLINE

314 explodes single branch MeSH terms.

315 There were two query sets where one platform failed to return any results. In sets \#024 and \#028,

316 Web of Science/MEDLINE returned 0 results across all twelve months, even though the syntax

317 of the queries were correct and one of the queries had returned results in a pilot test but then

318 dropped them in subsequent tests. Additionally, in sets \#025, \#027, and \#029, the Web of

319 Science/MEDLINE search result counts were initially within a reasonable range of the other

320 platforms in the respective sets, but then diverged substantially. For example, in search set \#025, 
321 Web of Science/MEDLINE returned a maximum of 13021 records and a minimum of 12652

322 records from October to April. However, the same query returned a maximum of 629 records

323 and a minimum of 619 records from May to September, indicating a drop of over 12000 records

324 for the same search query. In search set \#027, Web of Science/MEDLINE returned search counts

325 that were different but comparable to the other four databases, but then in May again, the counts

326 increased by nearly 40000 records and remained within that range until the end of data

327 collection. For search set \#029, the search result counts were again within range of the other four

328 databases through April, but then in May and until the end of data collection, the query no longer

329 retrieved any records. The only pattern among these three queries was that the sudden changes in

330 search result counts occurred in May.

\section{Differences in Database Currency}

332 The time it takes to import the overall PubMed file among platforms also impacts the retrieval

333 sets. On January 24, 2020, the US National Library of Medicine recommended an interim search

334 strategy for searching PubMed for literature related to the Covid-19 virus [54]. Their

335 recommended search query searched all fields for the term 2019-nCoV or for the terms wuhan

336 and coronavirus in the title and abstract fields:

337 2019-nCoV[All Fields] OR (wuhan[tiab] AND coronavirus[tiab])

338 We modified the search strategy to use it among all platforms and queried the PubMed platforms

339 at irregular intervals. Results show that for the PubMed data file, generally, all of the platforms

340 return different results for the same query for new literature. Results also include three versions

341 of NLM's interface to PubMed. Two versions are for legacy PubMed but show result counts for

342 when records are sorted by Best Match or Most Recent, since legacy PubMed applied two

343 different sorting algorithms in this version of PubMed [31]. We also show search results for the 
344 new version of PubMed, which does not apply different sorting algorithms for Best Match or

345 Most Recent, but which does report different search counts than both legacy PubMed results. As

346 Figure 6 illustrates, these different platforms for PubMed retrieve newly added records at

347 different rates, likely because they receive and incorporate the PubMed data file at different

348 times (Figure 6).

349 [ Insert Figure 6 here ]

350 Fig. 6. Search result count differences for COVID-19 related searches across PubMed based

351 platforms. PubMed Legacy Most Recent Sort (PMLMR), PubMed Legacy Best Match Sort

352 (PMLBM), PubMed New (PMN), ProQuest PubMed (PrQPM), EBSCOhost PubMed (EPM),

353 Web of Science PubMed (WSPM), Ovid PubMed (OPM)

\section{Discussion}

355 MEDLINE is the National Library of Medicine's premier bibliographic database that contains

356 more than 25 million references to journal articles in life sciences with a concentration on

357 biomedicine. The subject scope of MEDLINE is biomedicine and health, broadly defined to

358 encompass those areas of the life sciences, behavioral sciences, chemical sciences, and

359 bioengineering needed by health professionals and others engaged in basic research and clinical

360 care, public health, health policy development, or related educational activities. MEDLINE also

361 covers life sciences vital to biomedical practitioners, researchers, and educators, including

362 aspects of biology, environmental science, marine biology, plant and animal science as well as

363 biophysics and chemistry [55].

\section{Methods and Results Reproducibility}

365 Overall, we found several issues that impact the unevenness of search results across these

366 platforms and therefore their use as reproducible scientific instruments. Due to differences in 
367 search fields across MEDLINE platforms, such as with the publication date field, in

368 developments in publishing, such as online first versions of articles versus volume and issues

369 number versions, in the ability of databases to behave consistently over time, and to differences

370 in updates of the source file across platforms, it is difficult to construct queries that perform

371 consistently alike across systems and to get results that are consistent across systems.

372 Specifically, we found that queries restricted by publication dates continue to return new records

373 years past the limit on the publication date range. Data from this study begin to provide some

374 explanation for this variance. First, the growth of “online first” publications seems to have

375 complicated the traditional bibliographic record for journal articles which, in part, relies on the

376 relationship between an individual article and its volume and issue. The inclusion or absence of

377 metadata elements such as these are perhaps resulting in the creation of multiple records. In some

378 cases, changes to the original record has meant changes to the MeSH indexing. Additionally,

379 although all platforms provide a search field or a way to limit search results by publication date,

380 not all do so at the same level of detail. While simple publication date searching may have been

381 sufficient in a print only age when there was only one publication date, it is not sufficient in an

382 age when articles are published multiple times, via electronic publication dates and via print

383 dates with volumes and issues. The implication of each of these examples is that records risk

384 being dropped or added to time-restricted searches. Studies that rely on replicable search

385 strategies are at risk of being inherently flawed.

386 We further found that queries were more likely to return comparable search result counts when

387 they included multiple and specific field terms, such as queries combining keywords appearing

388 in a journal title or an article title, with an author name, and a relevant MeSH term (e.g., search

389 set \#013 and \#017). Practically speaking, this finding indicates that search results may be more 
uniform across platforms when searching for a known set of articles using a highly specific,

391 multi-faceted search query. Conversely, simple queries using just one or two keywords or MeSH

392 terms appear more susceptible to significant variations across platforms, underscoring the

393 continued importance of advanced training in literature database searching and consultation with

394 trained information professionals.

395 However, some platforms appear to be simply broken because they are not able to handle

396 exploding the MeSH hierarchy similarly (e.g., EBSCOhost and Web of Science outliers in search

397 set \#008), or they drop records from one month to the next even though the query has not been

398 altered. The lack of discernible causes of significant variance in search result counts over time

399 makes it impossible to adjust for such variance and undermines the trust in using bibliographic

400 databases to inform data-driven decision making.

401 Our longitudinal study suggested that some differences might be attributed to delays in

402 transferring the MEDLINE file to the vendors, since PubMed updates MEDLINE daily but the

403 other vendors may receive that update and then add that update at later dates. To test this, we ran

404 a COVID-19 search based on a query provided by the NLM in January 2020 and found that there

405 were uneven search result hits for new literature on the COVID-19 pandemic across platforms.

406 Although some of the differences in search result counts might be explained by the previous

407 issues, the main explanation here is likely due to delays in receiving and incorporating the

408 PubMed updates to the vendors. This suggests that if researchers need urgent access to the timely

409 publications, they should be concerned about which version of PubMed they use to collect data.

\section{Conclusion}

411 Remarkably, these results suggest we may be able to level one of the early critiques of Google

412 Scholar, which was its inability to replicate results from the same search over periods of time 
413 [56], on MEDLINE. What followed with research on Google Scholar were several studies

414 recommending against using Google Scholar as the sole database for systematic reviews

$415[35,57,58]$. If this criticism is valid for the MEDLINE platforms, our results may strengthen the

416 recommendation by Cochrane [23] that no single MEDLINE platform should be considered a

417 sole source.

418 The MEDLINE data is licensed to multiple vendors of information services who provide access

419 to the database on their platforms, such as EBSCOhost, ProQuest, Ovid, and Web of Science.

420 Any of these platforms are used by information specialists, health science librarians, medical

421 researchers, and others to conduct research, such as systematic reviews, in the biomedical

422 sciences. Our research examines results based on 29 sets of 145 carefully constructed search

423 queries, plus queries related to the COVID-19 pandemic, across these platforms and indicates

424 that these platforms provide uneven access to the literature, and thus depending on the platform

425 used, the validity of research based on the data gathered from them may be affected. Additional

426 research is required to understand other search related differences among these platforms,

427 including differences among the records that are retrieved, and how they specifically impact

428 research designs like systematic reviews and other biomedical research, and scientific

429 conclusions based on these studies. 


\section{References}

1. Hine C. Databases as scientific instruments and their role in the ordering of scientific work. Soc Stud Sci. 2006;36: 269-298.

2. Palmer CL, Cragin MH. Scholarship and disciplinary practices. Annu Rev Inf Sci Technol. 2008;42: 163-212.

3. Sampson M, Barrowman NJ, Moher D, Klassen TP, Pham B, Platt R, et al. Should metaanalysts search Embase in addition to Medline? J Clin Epidemiol. 2003;56: 943-955. doi:10.1016/S0895-4356(03)00110-0

4. Dunn K, Marshall JG, Wells AL, Backus JE. Examining the role of MEDLINE as a patient care information resource: an analysis of data from the Value of Libraries study. J Med Libr Assoc JMLA. 2017;105: 336. doi:10.5195/jmla.2017.87

5. Bezemer T, Groot MCH de, Blasse E, Berg MJ ten, Kappen TH, Bredenoord AL, et al. A Human(e) Factor in Clinical Decision Support Systems. J Med Internet Res. 2019;21: e11732. doi:10.2196/11732

6. McCain KW. Sources of polysemy in indexing practice: The case of games, experimental in MeSH. Proc Am Soc Inf Sci Technol. 2014;51: 1-4. doi:10.1002/meet.2014.14505101109

7. Hou J, Yang X, Chen C. Emerging trends and new developments in information science: a document co-citation analysis (2009-2016). Scientometrics. 2018;115: 869-892. doi:10.1007/s11192-018-2695-9

8. Hjørland B. Classical databases and knowledge organization: A case for boolean retrieval and human decision-making during searches. J Assoc Inf Sci Technol. 2015;66: 1559_ 1575. doi:10.1002/asi.23250

9. Beyer FR, Wright K. Can we prioritise which databases to search? A case study using a systematic review of frozen shoulder management. Health Inf Libr J. 2013;30: 49-58. doi:10.1111/hir.12009

10. Bramer WM, Giustini D, Kramer BM. Comparing the coverage, recall, and precision of searches for 120 systematic reviews in Embase, MEDLINE, and Google Scholar: a prospective study. Syst Rev. 2016;5: 39. doi:10.1186/s13643-016-0215-7

11. Rathbone J, Carter M, Hoffmann T, Glasziou P. A comparison of the performance of seven key bibliographic databases in identifying all relevant systematic reviews of interventions for hypertension. Syst Rev. 2016;5: 27. doi:10.1186/s13643-016-0197-5

12. Shariff SZ, Sontrop JM, Iansavichus AV, Haynes RB, Weir MA, Gandhi S, et al. Availability of renal literature in six bibliographic databases. Nephrol Dial Transplant Plus. 2012;5: 610-617. doi:10.1093/ckj/sfs152 
13. Boruff JT, Thomas A. Integrating evidence-based practice and information literacy skills in teaching physical and occupational therapy students. Health Inf Libr J. 2011;28: 264-272. doi:10.1111/j.1471-1842.2011.00953.x

14. Damarell RA, Tieman JJ. Searching PubMed for a broad subject area: how effective are palliative care clinicians in finding the evidence in their field? Health Inf Libr J. 2016;33: 49-60. doi:10.1111/hir.12120

15. Ondrusek AL, Ren X, Yang C. A Content Analysis of Strategies and Tactics Observed Among MLIS Students in an Online Searching Course. J Educ Libr Inf Sci. 2017 [cited 5 Dec 2019]. doi:10.3138/jelis.58.3.141

16. Young S, Duffull S. A learning-based approach for performing an in-depth literature search using MEDLINE. J Clin Pharm Ther. 2011;36: 504-512. doi:10.1111/j.13652710.2010.01204.x

17. Clarke RI. Breaking records: The history of bibliographic records and their influence in conceptualizing bibliographic data. Cat Classif Q. 2015;53: 286-302. doi:10.1080/01639374.2014.960988

18. Darmoni SJ, Soualmia LF, Letord C, Jaulent M-C, Griffon N, Thirion B, et al. Improving information retrieval using Medical Subject Headings Concepts: a test case on rare and chronic diseases. J Med Libr Assoc JMLA. 2012;100: 176. doi:10.3163/15365050.100.3.007

19. Amrhein V, Korner-Nievergelt F, Roth $\mathrm{T}$. The earth is flat ( $\mathrm{p}>0.05)$ : significance thresholds and the crisis of unreplicable research. PeerJ. 2017;5: e3544. doi:10.7717/peerj.3544

20. Baker M. 1,500 scientists lift the lid on reproducibility. Nat News. 2016;533: 452. doi:10.1038/533452a

21. Collaboration OS. Estimating the reproducibility of psychological science. Science. 2015;349: aac4716. doi:10.1126/science.aac4716

22. Moher D, Liberati A, Tetzlaff J, Altman DG, Group TP. Preferred Reporting Items for Systematic Reviews and Meta-Analyses: The PRISMA Statement. PLOS Med. 2009;6: e1000097. doi:10.1371/journal.pmed.1000097

23. Cochrane Handbook for Systematic Reviews of Interventions. [cited 2 Apr 2020]. Available: /handbook

24. Savoy J. Bibliographic database access using free-text and controlled vocabulary: an evaluation. Inf Process Manag. 2005;41: 873-890.

25. Tenopir C. Full text database retrieval performance. Online Rev. 1985;9: 149-164. doi:10.1108/eb024180 
26. Dunikowski LG. EMBASE and MEDLINE searches. Can Fam Physician. 2005;51: 1191. Available: https://www.ncbi.nlm.nih.gov/pmc/articles/PMC1479462/

27. Bethel A, Rogers M. A checklist to assess database-hosting platforms for designing and running searches for systematic reviews. Health Inf Libr J. 2014;31: 43-53. doi:10.1111/hir.12054

28. Rethlefsen M, Ayala AP, Kirtley S, Koffel J, Waffenschmidt S. PRISMA-S: PRISMA Search Reporting Extension. 2019 [cited 2 Apr 2020]. doi:10.17605/OSF.IO/YGN9W

29. Allison MM. Comparison of cinahl ${ }^{\circledR}$ via EBSCOhost ${ }^{\circledR}$, OVID $\AA$, and ProQuest ${ }^{\circledR}$. J Electron Resour Med Libr. 2006;3: 31-50. doi:10.1300/j383v03n01_04

30. Craven J, Jefferies J, Kendrick J, Nicholls D, Boynton J, Frankish R. A comparison of searching the Cochrane library databases via CRD, Ovid and Wiley: implications for systematic searching and information services. Health Inf Libr J. 2014;31: 54-63. doi:10.1111/hir.12046

31. Collins M. Updated Algorithm for the PubMed Best Match Sort Order. NLM Technical Bulletin; 2017. Available: https://www.nlm.nih.gov/pubs/techbull/jf17/jf17_pm_best_match_sort.html

32. Lam MT, McDiarmid M. Increasing number of databases searched in systematic reviews and meta-analyses between 1994 and 2014. J Med Libr Assoc. 2016;104: 284-289. doi:10.5195/jmla.2016.141

33. Toews LC. Compliance of systematic reviews in veterinary journals with Preferred Reporting Items for Systematic Reviews and Meta-Analysis (PRISMA) literature search reporting guidelines. J Med Libr Assoc. 2017;105: 233-239. doi:10.5195/jmla.2017.246

34. Vassar M, Yerokhin V, Sinnett PM, Weiher M, Muckelrath H, Carr B, et al. Database selection in systematic reviews: an insight through clinical neurology. Health Inf Libr J. 2017;34: 156-164. doi:10.1111/hir.12176

35. Bramer WM, Giustini D, Kramer BM, Anderson P. The comparative recall of Google Scholar versus PubMed in identical searches for biomedical systematic reviews: a review of searches used in systematic reviews. Syst Rev. 2013;2: 115.

36. Lemeshow AR, Blum RE, Berlin JA, Stoto MA, Colditz GA. Searching one or two databases was insufficient for meta-analysis of observational studies. J Clin Epidemiol. 2005;58: 867-873. doi:10.1016/j.jclinepi.2005.03.004

37. Yoshii A, Plaut DA, McGraw KA, Anderson MJ, Wellik KE. Analysis of the reporting of search strategies in Cochrane systematic reviews. J Med Libr Assoc. 2009;97: 21-29. doi:10.3163/1536-5050.97.1.004 
38. Ahmadi M, Sarabi RE, Orak RJ, Bahaadinbeigy K. Information Retrieval in Telemedicine: a Comparative Study on Bibliographic Databases. Acta Inform Medica. 2015;23: 172-176. doi:10.5455/aim.2015.23.172-176

39. Mowshowitz A, Kawaguchi A. Bias on the web. Commun ACM. 2002 [cited 2 Apr 2020]. Available: https://dl.acm.org/doi/abs/10.1145/567498.567527

40. Vaughan L, Thelwall M. Search engine coverage bias: evidence and possible causes. Inf Process Manag. 2004;40: 693-707. doi:10.1016/S0306-4573(03)00063-3

41. Buchanan S, Salako A. Evaluating the usability and usefulness of a digital library. Libr Rev. 2009;58: 638-651. doi:10.1108/00242530910997928

42. Edwards A, Kelly D, Azzopardi L. The Impact of Query Interface Design on Stress, Workload and Performance. In: Hanbury A, Kazai G, Rauber A, Fuhr N, editors. Advances in Information Retrieval. Springer International Publishing; 2015. pp. 691-702.

43. Ho GJ, Liew SM, Ng CJ, Shunmugam RH, Glasziou P. Development of a Search Strategy for an Evidence Based Retrieval Service. PLOS ONE. 2016;11: e0167170. doi:10.1371/journal.pone.0167170

44. Peng RD. Reproducible research and Biostatistics. Biostatistics. 2009;10: 405-408. doi:10.1093/biostatistics/kxp014

45. Boddy K, Younger P. What a difference an interface makes: just how reliable are your search results? Focus Altern Complement Ther. 2009;14: 5-7. doi:10.1111/j.20427166.2009.tb01854.x

46. Younger P, Boddy K. When is a search not a search? A comparison of searching the amed complementary health database via EBSCOhost, OVID and DIALOG. Health Inf Libr J. 2009;26: 126-135. doi:10.1111/j.1471-1842.2008.00785.x

47. Haase A, Follmann M, Skipka G, Kirchner H. Developing search strategies for clinical practice guidelines in SUMSearch and Google Scholar and assessing their retrieval performance. BMC Med Res Methodol. 2007;7: 28. doi:10.1186/1471-2288-7-28

48. Hill B. Comparison of journal title coverage between CINAHL and Scopus. J Med Libr Assoc JMLA. 2009;97: 313.

49. Nourbakhsh E, Nugent R, Wang H, Cevik C, Nugent K. Medical literature searches: a comparison of PubMed and Google Scholar. Health Inf Libr J. 2012;29: 214-222. doi:10.1111/j.1471-1842.2012.00992.x

50. Goodman SN, Fanelli D, Ioannidis JPA. What does research reproducibility mean? Sci Transl Med. 2016;8: 341ps12-341ps12. doi:10.1126/scitranslmed.aaf5027 
51. U.S. National Library of Medicine. The New PubMed is Here. U.S. National Library of Medicine; 2020 [cited 15 Apr 2020]. Available:

https://www.nlm.nih.gov/pubs/techbull/nd19/nd19_pubmed_new.html

52. Advanced PubMed Searching Resource Packet. U.S. National Library of Medicine; 2017. Available: https://nnlm.gov/sites/default/files/shared/files/trifolds/pubmedpacket.pdf

53. Thomas S, Walsh D, Aktas A. Systematic bias in cancer patient-reported outcomes: symptom 'orphans' and 'champions.' BMJ Support Palliat Care. 2019;9: 67-74. doi:10.1136/bmjspcare-2014-000835

54. New MeSH Supplementary Concept Record for the 2019 Novel Coronavirus, Wuhan, China. NLM Technical Bulletin. 2020 Jan-Feb. U.S. National Library of Medicine; 2020. Available: https://www.nlm.nih.gov/pubs/techbull/jf20/brief/jf20_mesh_novel_coronavirus.html?utm_ medium=email\&utm_source=govdelivery

55. MEDLINE®: Description of the Database. U.S. National Library of Medicine; 2019. Available: https://www.nlm.nih.gov/bsd/medline.html

56. Jacsó P. Google Scholar: the pros and the cons. Online Inf Rev. 2005;29: 208-214.

57. Gehanno J-F, Rollin L, Darmoni S. Is the coverage of google scholar enough to be used alone for systematic reviews. BMC Med Inform Decis Mak. 2013;13: 7. doi:10.1186/14726947-13-7

58. Giustini D, Boulos MNK. Google Scholar is not enough to be used alone for systematic reviews. Online J Public Health Inform. 2013;5: 214. doi:10.5210/ojphi.v5i2.4623 
PubMed, Search 003

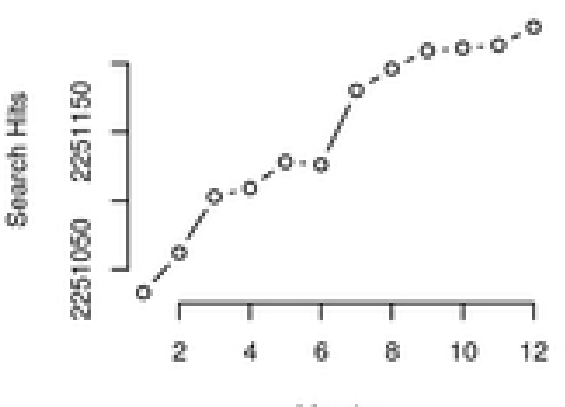

Months

PubMed, Search 004

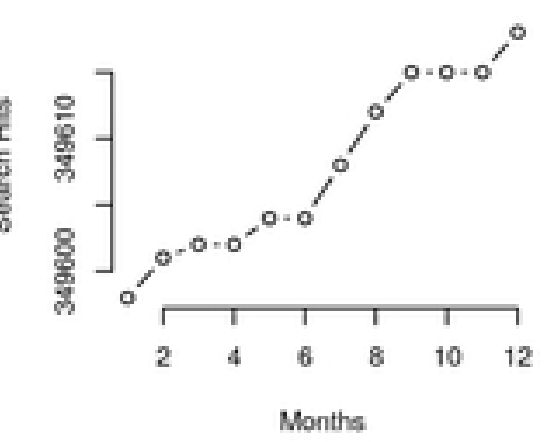

PubMed, Search 005

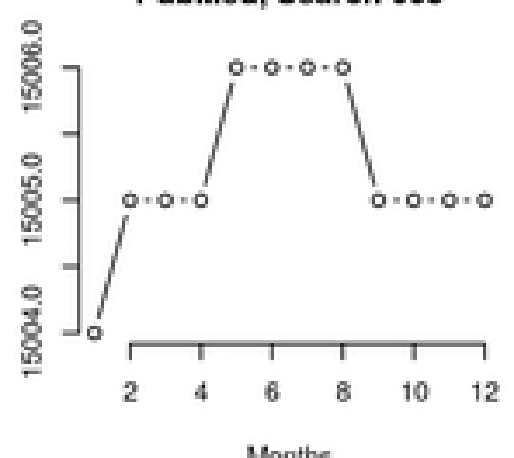

Months

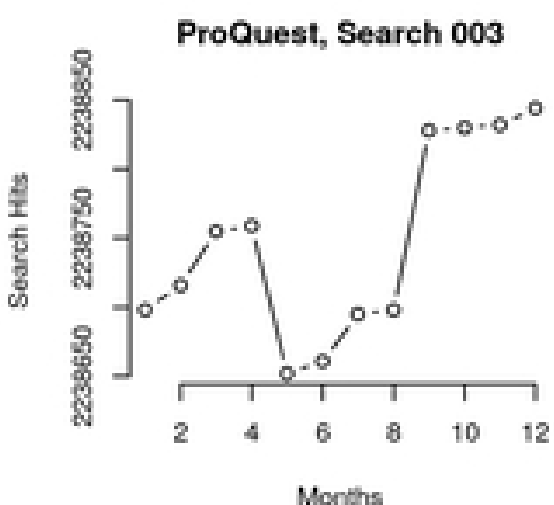

ProQuest, Search 004

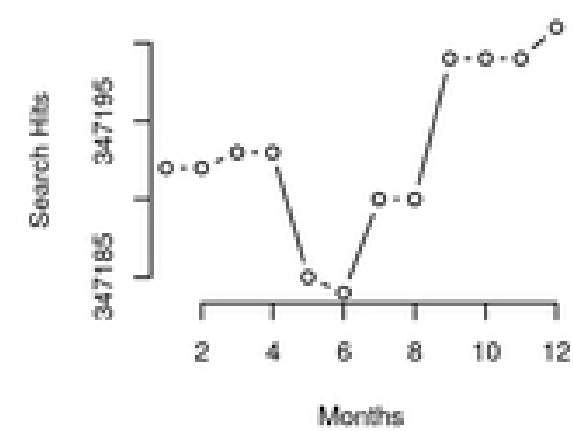

ProQuest, Search 005

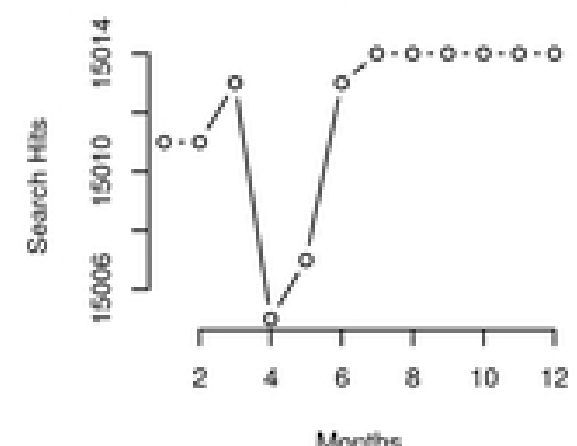

EBSCOhost, Search 003

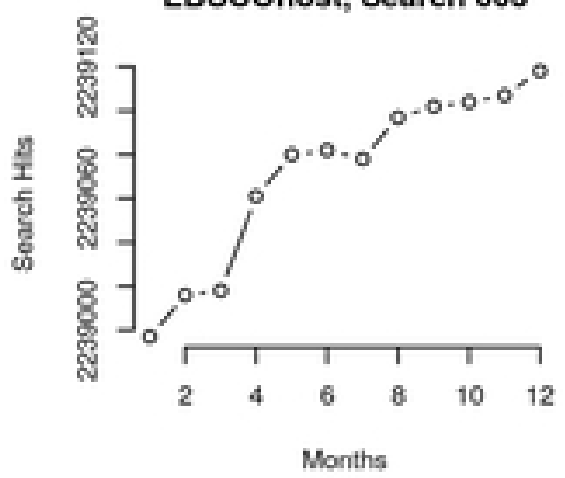

EBSCOhost, Search 004

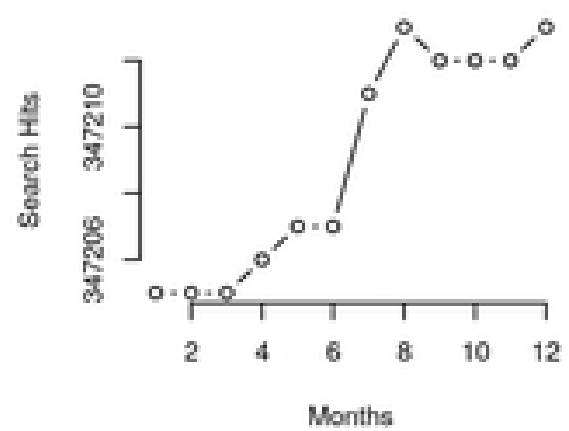

EBSCOhost, Search 005

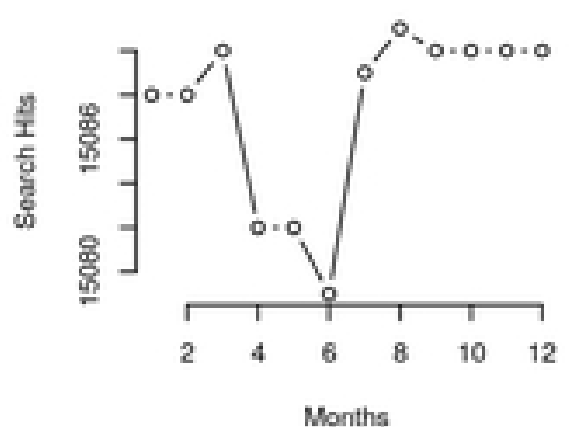

Web of Science, Search 003

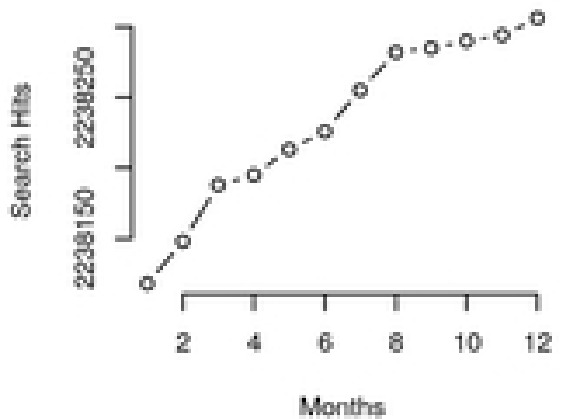

Web of Science, Search 004

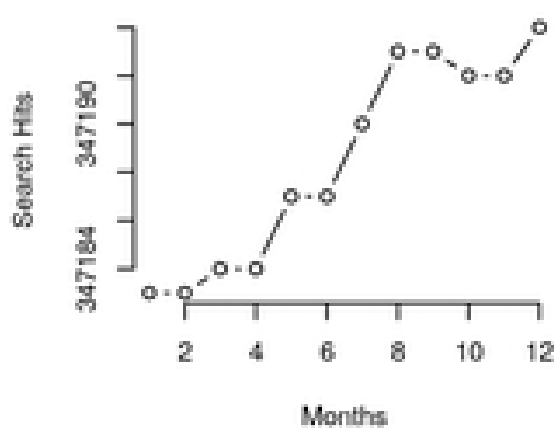

Web of Science, Search 005

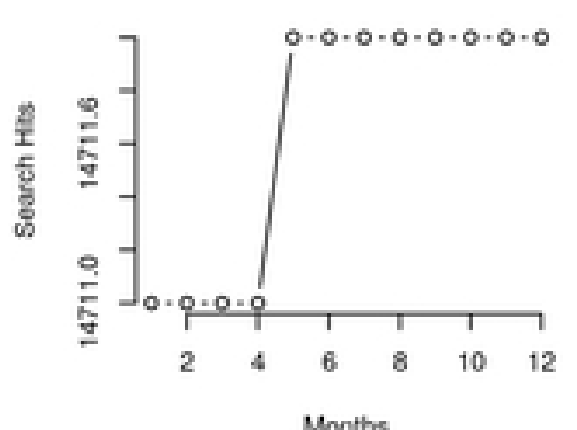

Ovid, Seareh 003

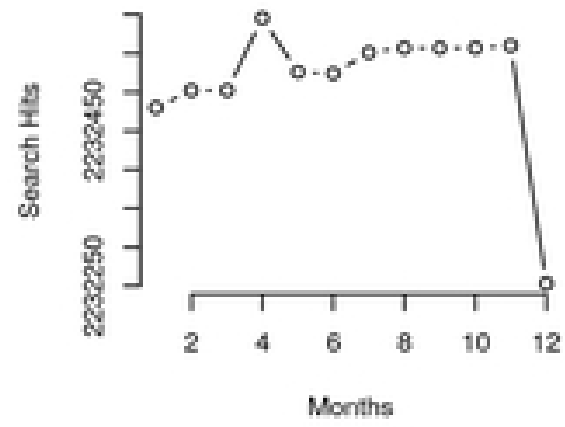

Ovid, Search 004

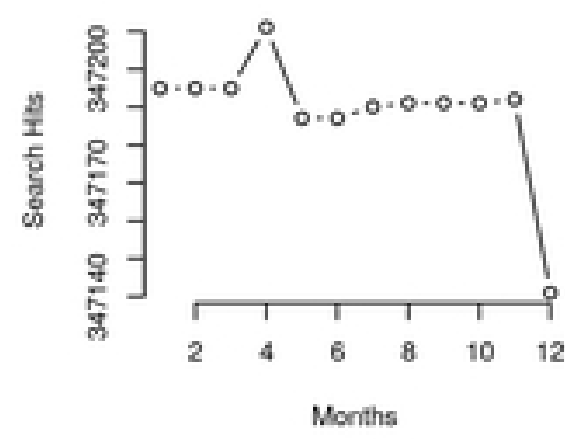

Ovid, Search 005

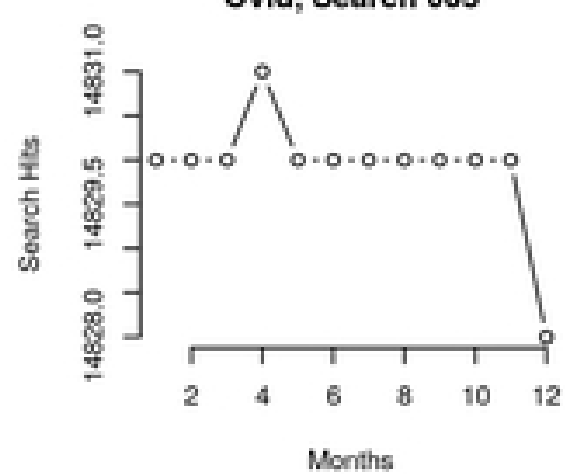

Figure 2 
PubMed, Search 009

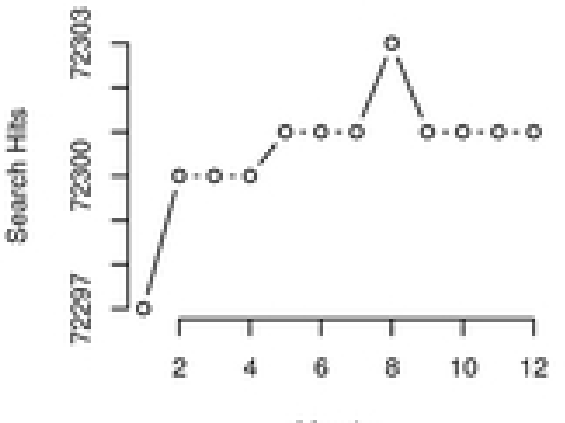

Months

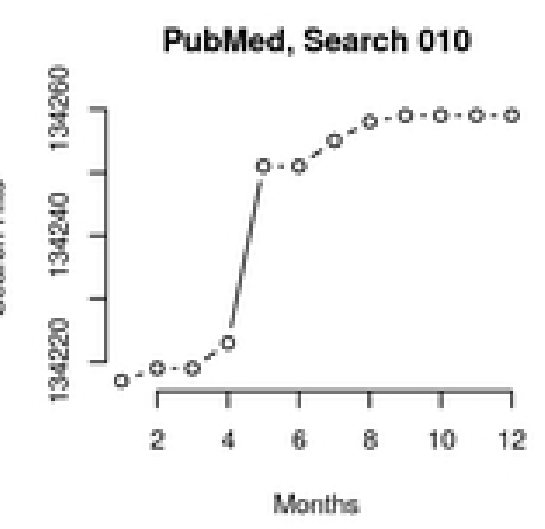

PubMed, Search 011

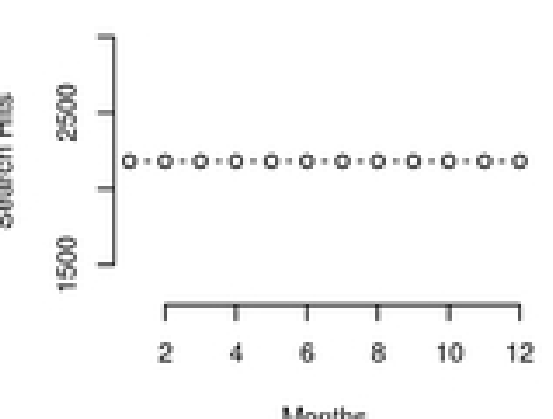

Months
ProQuest, Search 009

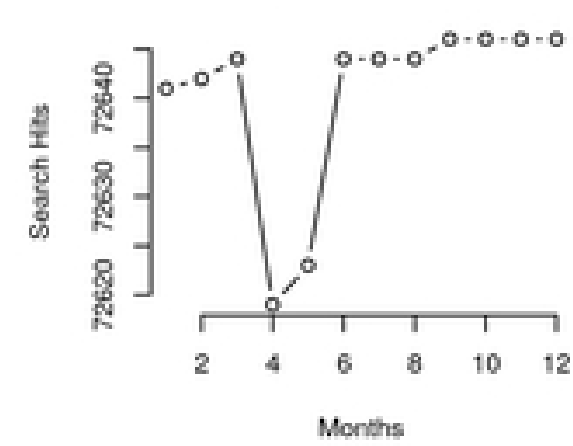

ProQuest, Search 010

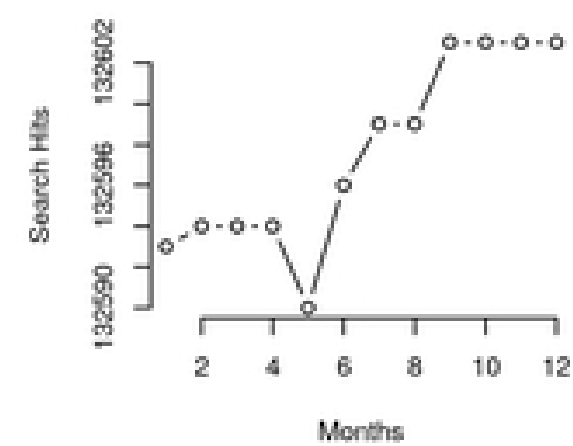

ProQuest, Search 011

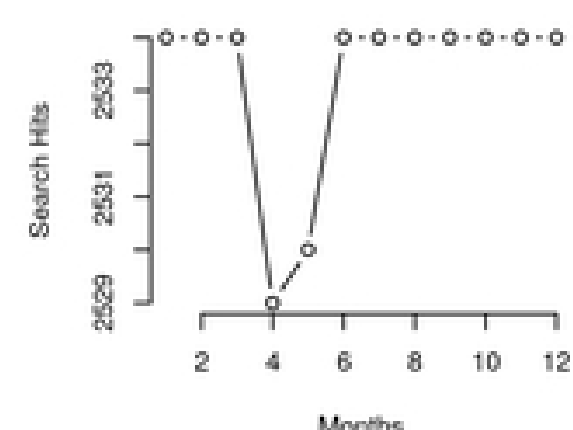

EBSCOhost, Seareh 009

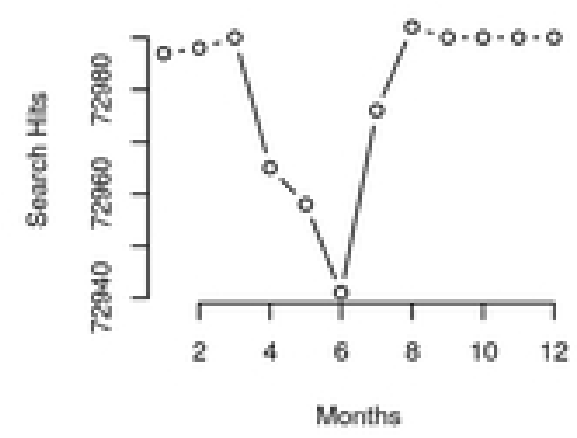

EBSCOhost, Seareh 010

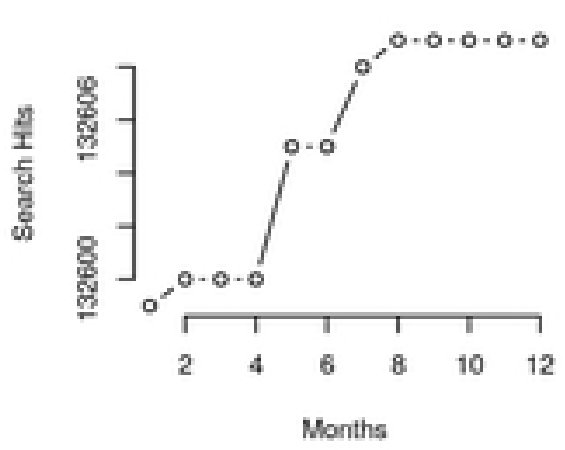

EBSCOhost, Seareh 011

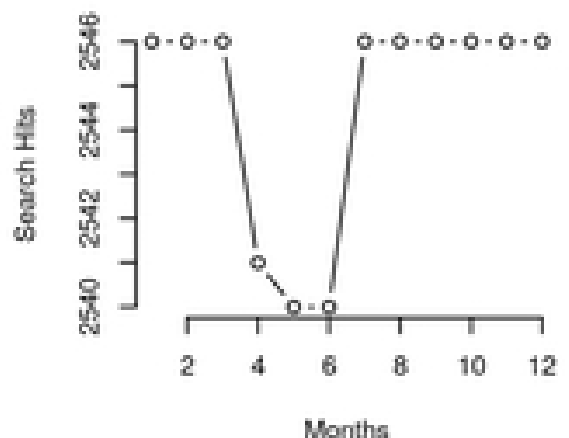

Web of Science, Search 009

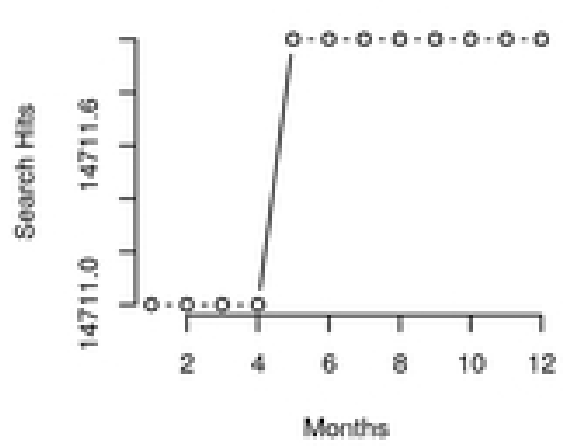

Web of Science, Search 010

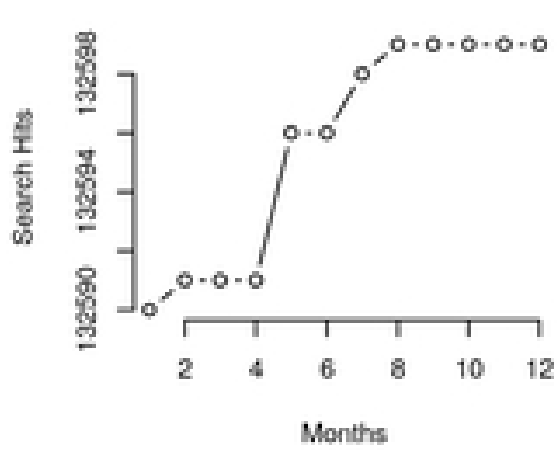

Web of Science, Search 011

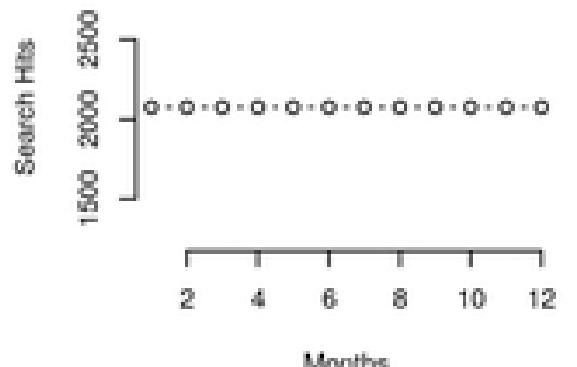

Ovid, Seareh 009

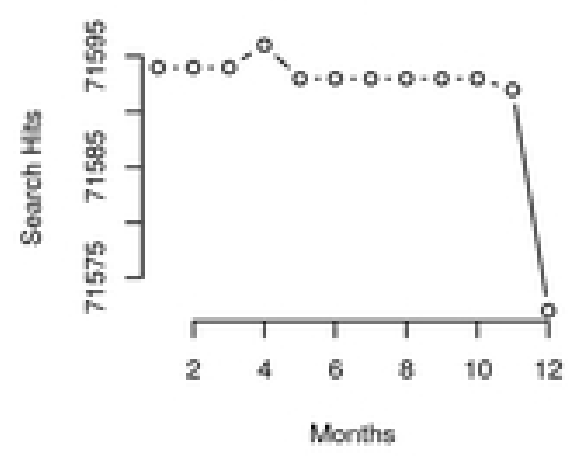

Ovid, Search 010

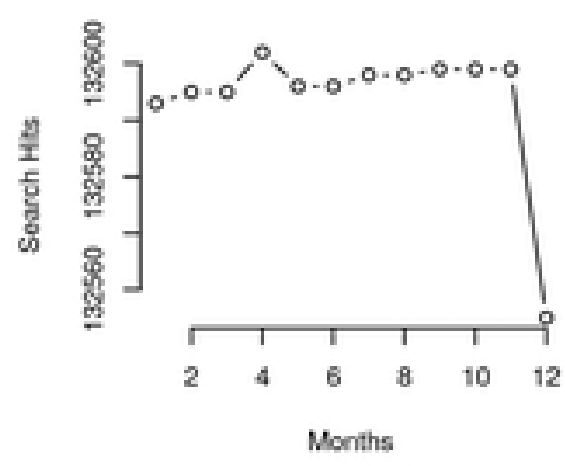

Ovid, Search 011

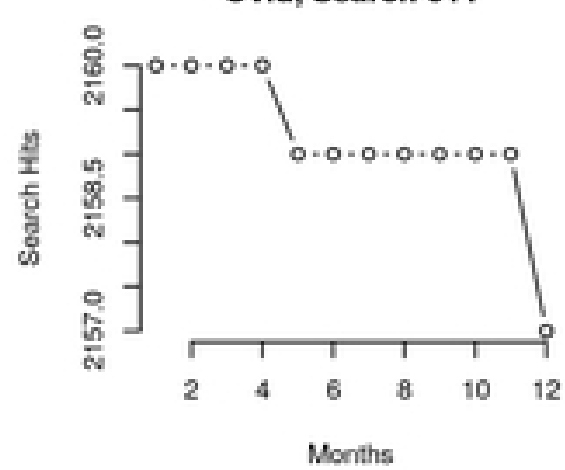

Figure 4 
